\title{
A New Robust Decentralized Control Method for Interconnected Nonlinear Systems Based on State Extension and Adaptive Tracking
}

\author{
GU Zhifeng ${ }^{1, a}$, WANG Yutong ${ }^{1}$, YANG Guixin ${ }^{2}$ and CAO Man ${ }^{1}$ \\ ${ }^{1}$ Vehicles and Electrical department, Mechanical Engineering College, Shi Jiazhuang, China \\ ${ }^{2} 54^{\text {th }}$ Research Institute, China Electronics Technology Corporation, Shi Jiazhuang, China
}

Keywords: state extension and tracking; interconnected nonlinear system; robust decentralized control; decentralized L2 gain disturbance attenuation; state adaptive tracking.

Abstract. In this paper, a new nonlinear robust adaptive decentralized control for a class of large-scale interconnected system (LS-Inter-SYS) with the uncertain parameters and disturbances is proposed. By using the mean-value theorem of the multivariate function, the cross terms and the uncertain parts of the LS-Inter-SYS are extended to the new state variables and adaptive tracking estimated, then the LS-Inter-SYS can be divided into separated subsystems. Simulation results show that the new decentralized robust adaptive control method is effective and has the characteristics of universality, simplicity and rapidity by introducing the K-Class functions and additional control variable.

\section{Introduction}

Because of the large scale, the high nonlinear, and the complicated structure, the nonlinear robust control of the large-scale interconnected system (LS-Inter-SYS), such as power system, aerospace system, robot system, chemical applications and telecommunication networks, etc., has attracted considerable attention $^{[1]-[6]}$. In the LS-Inter-SYS, the subsystems often affect each other. When the distance between the subsystems is long, it is very difficult and complicated for the conventional centralized controller to collect the large status information. So it is necessary to realize the decentralized robust control of the LS-Inter-SYS. The decentralized control has the characteristics of avoiding the computational complexity, reducing economical costs involved in collecting information exchange among several distant systems, and enhancing the robustness when the interaction among, and guaranteeing the local system to be safe and operate at a failure mode.

In [3], one decentralized L2-gain attenuation control method is presented, but in the considered class of LS-Inter-SYS, any two subsystems are interacted only via their outputs, note their complete states. The decentralized $\mathrm{H}_{\infty}$ control method of LS-Inter-SYS with uncertainties and disturbances is studied in [4]-[5], but only the interconnected terms or parts of the LS-Inter-SYS are nonlinear, other parts is linear. These problems are also apparent in [6]-[8]. The adaptive control of the nonlinear LS-Inter-SYS is presented in [9]-[11]. 
Motivated by the above observation, in this paper, for a class of the universal LS-Inter-SYS with uncertain parameters and disturbances, a new recursive decentralized control method is presented. In the new L2-gain attenuation back-stepping adaptive control (L2-BSAC) method, because the K-Class functions and additional control variable are adopted, the state parameters convergence speed will be improved, and the calculation of the L2-gain attenuation will be simple.

\section{Nonlinear robust decentralized control based on mean-value theorem and back-stepping}

Considering the following interconnected system with the uncertain parameter and the disturbance:

$$
\begin{gathered}
\dot{x}_{1}=-x_{1}+\cos \left(2 x_{1}+2 x_{2}\right)+x_{2} \bar{\theta}_{1}+u_{1}+\varepsilon_{1} \\
\dot{x}_{2}=-3 x_{2}+\sin \left(x_{1}+x_{2}\right)+x_{1} \bar{\theta}_{2}+u_{2}+\varepsilon_{2} \\
\boldsymbol{y}=\left[y_{1}, y_{2}\right]^{\mathrm{T}}=\left[x_{1}, x_{2}\right]^{\mathrm{T}}
\end{gathered}
$$

Where $\bar{\theta}_{1}$ and $\bar{\theta}_{2}$ are the uncertain parameters; $\varepsilon_{1}$ and $\varepsilon_{2}$ are the limited-band white noise.

We know the mean-value theorem of the multivariate function can be shown as:

$$
f(\boldsymbol{X})=f\left(\boldsymbol{X}_{1}\right)+\nabla f\left(\boldsymbol{X}_{0}\right)\left(\boldsymbol{X}_{2}-\boldsymbol{X}_{1}\right)
$$

Where $f(\boldsymbol{X})$ is the smooth function, $\boldsymbol{X}=\left[x_{1}, \cdots, x_{n}\right]^{\mathrm{T}} \in R^{n} ; \nabla f\left(\boldsymbol{X}_{0}\right)$ is the gradient of the $f(\boldsymbol{X})$ at $\boldsymbol{X}_{0}, \boldsymbol{X}_{0} \in\left[X_{1}, X_{2}\right]$.

By the mean-value theorem of the multivariate function, we will transform the system (1) into:

$$
\begin{gathered}
\dot{x}_{1}=-x_{1}+\cos \left(2 x_{1}\right)+\theta_{1}+u_{1}+\varepsilon_{1} \\
\dot{x}_{2}=-3 x_{2}+\sin \left(x_{2}\right)+\theta_{2}+u_{2}+\varepsilon_{2}
\end{gathered}
$$

Where $0<\sigma_{1}<1,0<\sigma_{2}<1, \theta_{1}=\sin \left(2 x_{1}+2 \sigma_{1} x_{2}\right) x_{2}+x_{2} \bar{\theta}_{1}$ and $\theta_{2}=\cos \left(\sigma_{2} x_{1}+x_{2}\right) x_{1}+x_{1} \bar{\theta}_{2}$.

Defining $V_{1}=x_{1}^{2} / 2+\tilde{\theta}_{1}^{2} /\left(2 \rho_{1}\right)$, by (3.1), yield:

$$
\dot{V}_{1}=x_{1} \dot{x}_{1}+\tilde{\theta}_{1} \dot{\tilde{\theta}}_{1} / \rho_{1}=x_{1}\left(-x_{1}+\cos \left(2 x_{1}\right)+\theta_{1}+u_{1}+\varepsilon_{1}\right)+\tilde{\theta}_{1} \dot{\tilde{\theta}}_{1} / \rho_{1}
$$

Where $\hat{\theta}_{1}=\theta_{1}-\widetilde{\theta}_{1}$ is the estimated value of $\theta_{1}, \widetilde{\theta}_{1}$ is the estimation error.

By (4), we can obtain the control law and the adaptive law as follows:

$$
\begin{gathered}
u_{1}=x_{1}-\cos \left(2 x_{1}\right)-\hat{\theta}_{1}-m_{1} x_{1}+u_{f 1} \\
\dot{\hat{\theta}}_{1}=\rho_{1} x_{1}
\end{gathered}
$$

Where $m_{1}=f_{1}\left(\left|x_{1}\right|\right)+c_{1}>0$ is the K-Class function, $u_{f 1}$ is the additive control variable.

Substituting (5) and (6) into (4), we obtain:

$$
\begin{gathered}
\dot{V}_{1}=-m_{1} x_{1}^{2}+x_{1} \varepsilon_{1}+x_{1} u_{f 1} \\
H_{1}=\dot{V}_{1}+\left(x_{1}^{2}-\gamma_{1}^{2} \varepsilon_{1}^{2}\right) / 2
\end{gathered}
$$

When $u_{f 1}=-\frac{x_{1}\left(1+\gamma_{1}^{2}\right)}{2 \gamma_{1}^{2}}$ is substituted into (5) and (8), we obtain:

$$
\begin{aligned}
H_{1} & =-m_{1} x_{1}^{2}-\left(\gamma_{1} \varepsilon_{1} / \sqrt{2}-x_{1} / \sqrt{2} \gamma_{1}\right)^{2} \leq 0 \\
u_{1} & =\left[1-m_{1}-\left(1+\gamma_{1}^{2}\right) / 2 \gamma_{1}^{2}\right] x_{1}-\cos \left(2 x_{1}\right)-\hat{\theta}_{1}
\end{aligned}
$$

Because the integral of $H_{1}=\dot{V}_{1}+\left(\left\|y_{1}(t)\right\|^{2}-\gamma_{1}^{2}\left\|\varepsilon_{1}(t)\right\|^{2}\right) / 2 \leq 0$ is $\left\|\mathrm{y}_{1}(t)\right\|^{2} \leq \gamma_{1}^{2}\left\|\varepsilon_{1}(t)\right\|^{2}+V_{1}(0),(6)$ and $(10)$ can realize the nonlinear robust adaptive L2-gain attenuation control of the system (1.1).

Defining $V_{2}=x_{2}^{2} / 2+\tilde{\theta}_{2}^{2} /\left(2 \rho_{1}\right)$, by (3.2), we obtain: 


$$
\dot{V}_{2}=x_{2} \dot{x}_{2}+\tilde{\theta}_{2} \dot{\tilde{\theta}}_{2} / \rho_{1}=x_{2}\left(-3 x_{2}+\sin \left(x_{2}\right)+\hat{\theta}_{2}+\tilde{\theta}_{2}+u_{2}+\varepsilon_{2}\right)+\tilde{\theta}_{2} \dot{\tilde{\theta}}_{2} / \rho_{1}
$$

Where $\hat{\theta}_{2}=\theta_{2}-\widetilde{\theta}_{2}$ is the estimated value of $\theta_{2}, \widetilde{\theta}_{2}$ is the estimation error.

By (11), we can obtain the control law and the adaptive law as follows:

$$
\begin{gathered}
u_{2}=3 x_{2}-\sin \left(x_{2}\right)-\hat{\theta}_{2}-m_{2} x_{2}+u_{f 2} \\
\dot{\hat{\theta}}_{2}=\rho_{2} x_{2}
\end{gathered}
$$

Where $m_{2}=f_{2}\left(\left|x_{2}\right|\right)+c_{2}>0$ is the K-Class function, $u_{f 2}$ is the additive control variable.

Substituting (12) and (13) into (11), we obtain:

$$
\begin{gathered}
\dot{V}_{2}=-m_{2} x_{2}^{2}+x_{2} \varepsilon_{2}+x_{2} u_{f 2} \\
H_{2}=-m_{2} x_{2}^{2}+x_{2} \varepsilon_{2}+x_{2} u_{f 2}+\left(x_{2}^{2}-\gamma_{2}^{2} \varepsilon_{2}^{2}\right) / 2
\end{gathered}
$$

When $u_{f 2}=-x_{2}\left(1+\gamma_{2}^{2}\right) /\left(2 \gamma_{2}^{2}\right)$ is substituted into (12) and (15), we obtain:

$$
\begin{gathered}
H_{2}=\dot{V}_{2}+\left(x_{2}^{2}-\gamma_{2}^{2} \varepsilon_{2}^{2}\right) / 2=-m_{2} x_{2}^{2}-\left(\gamma_{2} \varepsilon_{2} / \sqrt{2}-x_{2} / \sqrt{2} \gamma_{2}\right)^{2} \leq 0 \\
u_{2}=3 x_{2}-\sin \left(x_{2}\right)-\hat{\theta}_{2}-m_{2} x_{2}-x_{2}\left(1+\gamma_{2}^{2}\right) / 2 \gamma_{2}^{2}=\left[3-m_{2}-\left(1+\gamma_{2}^{2}\right) / 2 \gamma_{2}^{2}\right] x_{2}-\sin \left(x_{2}\right)-\hat{\theta}_{2}
\end{gathered}
$$

Because the integral of $H_{2}=\dot{V}_{2}+\left(\left\|y_{2}(t)\right\|^{2}-\gamma_{2}^{2}\left\|\varepsilon_{2}(t)\right\|^{2}\right) / 2 \leq 0$ is $\left\|y_{2}(t)\right\|^{2} \leq \gamma_{2}^{2}\left\|\varepsilon_{2}(t)\right\|^{2}+V_{2}(0)$, the control law (17) and the adaptive (13) can realize adaptive L2-gain acontrol of the system (1.2).

\section{Simulation}

For system (1), when $x_{1}$ and $x_{2}$ are substituted by $x_{1}-x_{1 d}$ and $x_{2}-x_{2 d}$ in (6), (10), (13), (17), $x_{1}$ and $x_{2}$ will track the instruction signal $x_{1 d}$ and $x_{2 d}$. When $\rho_{1}=\rho_{2}=10, \gamma_{1}=\gamma_{2}=1$, $x_{1 d}=20 \sin (10 \pi), m_{1}=50\left(x_{1}-x_{1 \mathrm{~d}}\right)^{2}=50 e_{1}^{2}, x_{2 d}=10 \sin (\pi \mathrm{t}), m_{2}=20\left(x_{2}-x_{2 \mathrm{~d}}\right)^{2}=20 e_{2}^{2}$, he curves of $x_{1}$, $x_{2}, u_{1}, u_{2}, \theta_{1}$ and $\theta_{2}$ are shown as Figure 1 and Figure 2 .

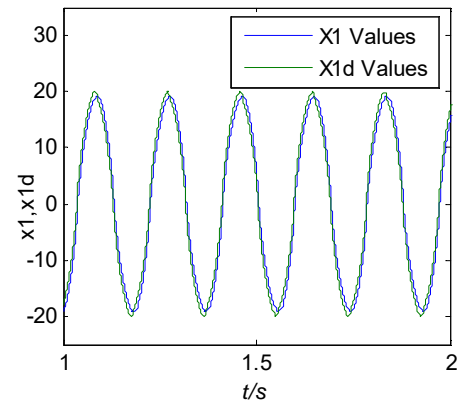

(a) $x_{1}, x_{1 \mathrm{~d}}$

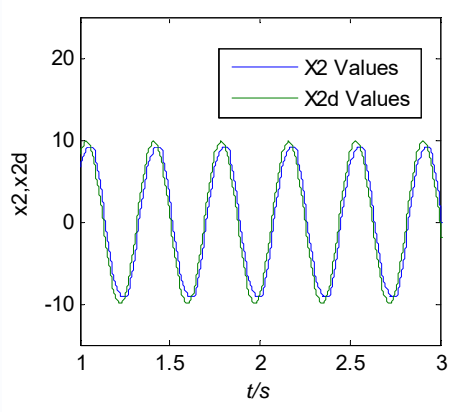

(b) $x_{2}, x_{2 \mathrm{~d}}$

Figure 1 the curves of the state variables 


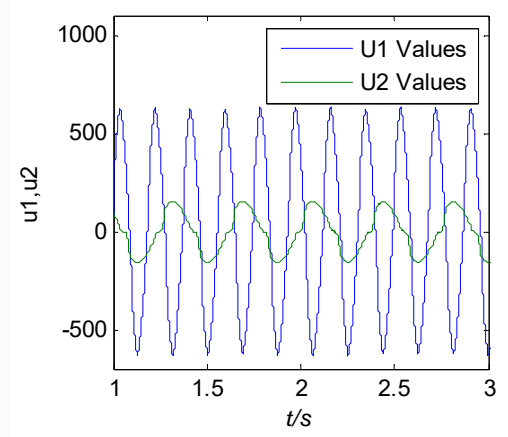

(a) $u_{1}, u_{2}$

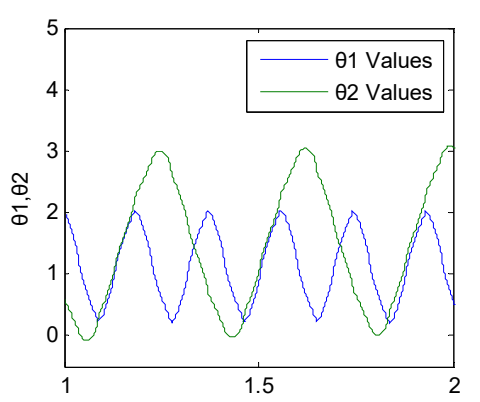

(b) $\theta_{1}, \theta_{2}$

Figure 2 the curves of the control and adaptive law

As Figure 1 and Figure 2 shown, when $\mathrm{m}_{1}$ and $\mathrm{m}_{2}$ are K-Class functions, $x_{1}$ and $x_{2}$ can track $x_{1 \mathrm{~d}}$ and $x_{2 \mathrm{~d}}$ more rapidly. The simulation results show that new control method can not only stabilize the interconnected system, adaptive to the uncertain parameters, but also attenuate the disturbance. Comparing with the traditional lower-triangular system structure and back-stepping method, the structure of the system (1) is more universal and the new control method has the characteristics of generality, simplicity, high efficiency and etc.

\section{Conclusions}

In this paper, a new recursive decentralized control method is presented. By using the mean-value theorem of the multivariate function, the cross terms and the uncertain parts of the LS-Inter-SYS are extended to the new state variables, the LS-Inter-SYS can be decomposed into many subsystems. For the converted subsystem, a new L2-gain attenuation back-stepping adaptive control (L2-BSAC) method is presented. In the new L2-BSAC, $m_{i, j}$ is the K-Class function, and the calculation process of the L2-gain attenuation is simplified by introducing into the new control variable. Simulation results show that the new control method can not only decentralized stabilize the large-scale interconnected system, adaptive to the uncertain parameters, but also attenuate the disturbances,.

\section{Acknowledgements}

This research is financially supported by National Natural Science Foundation of China (Grant No. 51407196).

\section{References}

[1] Gu Zhifeng, Zhu Changqing, Shao Tianzhang, etc. Robust Adaptive Control for the Excitation System Based on Total-State-Parameter Optimal Control. Control Theory \& Applications 2013; 30(7):856-862.

[2] ZhaoJing Wu, XueJun Xie, SiYing Zhang. Robust decentralized adaptive stabilization for a class of interconnected systems with unmodelled dynamics. International Journal of Systems Science 2004; 35(7):389-404.

[3] Zhongping Jiang, Daniel W. Repperger, David J.Hill. Decentralized Nonlinear Output-Feedback Stabilization With Disturbance Attenuation. IEEE Transactions On Automatic Control 2001; 46 (10): 16231629.

[4] Chen Ning, Gui Weihua. Robust Decentralized Hœ Control for Multi-Channel Systems with Norm-Bounded Parametric Uncertainties. Journal of Systems Engineering and Electronics 2007; 18(4):871-888.

[5] Zhao Zhihua, Chen Yuepeng, Zhang Qingling. Non-fragile decentralized Ho controller design for uncertain linear systems 2008; 19(2):321-328. 
[6] Fu Qin, Yang Chengwu. Robust Decentralized Control for a Class of Large-Scale Interconnected Nonlinear System with Mismatched Interconnections. ACTA Automatic Sinica 2008; 34(4): 488-4892.

[7] Ma Yuechao, Zhang Qingling. Decentralized Control for Nonlinear Large-Scale Interconnected Systems with Input Saturation. Control Theory \& Applications 2007; 24(4):683-686.

[8] Wu Jiancheng, Wo Songlin, Lu Guoping. Exponential Stability and Decentralized Control for Nonlinear Singular Large-scale Systems. Journal of Nanjing University of Science and Techno logy (Natural Science) 2009; 33(1):21-25.

[9] Chen mou, Jiang Changsheng, Chen Wenhua. Robust Sliding Mode Decentralized Control for a Class of Nonlinear Interconnected Large-Scale System With Neural Networks. Transactions of Nanjing University of Aeronautics \& Astronautics 2004; 21(4): 304-310.

[10] Huang Yishao, Zhou Dequn, Wang Zhengwu, etc.. Hळ Tracking-Based Decentralized Adaptive Output Feedback Fuzzy Control for a Class of Large-Scale MIMO Nonlinear System. Control and Decision 2010; 25 (11):1707-1713.

[11] Chen Weisheng, Li Junmin. Globally Stable Decentralized Adaptive Neural Network Back-Stepping Tracking Control. Control and Decision 2009; 24 (6):819-824. 